\title{
A NOTE ON MATRIX RICCATI SYSTEMS
}

\author{
W. J. COLES
}

1. Let $A(t)$ be an $n \times n$ matrix, continuous on an interval $I$; let $n_{1}, \cdots, n_{k}$ be positive integers such that $\sum_{i=1}^{k} n_{i}=n$. Let $A$ be partitioned into submatrices $A_{i j}$ which are $n_{i} \times n_{j},(i, j=1, \cdots, k)$; let $E_{m}$ be the identity matrix of order $m$; and let $A_{m}=\left(A_{m 1} \cdots A_{m k}\right)$. In this note we consider the matrix Riccati system with side condition

$$
Y^{\prime}=-Y A_{m} Y+A Y, \quad Y_{m}\left(t_{0}\right)=E_{n_{m}},
$$

where $Y=\operatorname{col}\left(Y_{1}, \cdots, Y_{k}\right)$ and $Y_{i}$ is $n_{i} \times n_{m}$. This equation is derived in a natural way as a generalization of the so-called Riccati system $[1 ; 2]$. Mainly we generalize some results of Levin [3], who treats the equation

$$
\Gamma^{\prime}=-\Gamma G_{3} \Gamma-\Gamma G_{4}+G_{1} \Gamma+G_{2},
$$

where $G_{1}, G_{2}, G_{3}$, and $G_{4}$ are $n_{1} \times n_{1}, n_{1} \times n_{2}, n_{2} \times n_{1}$, and $n_{2} \times n_{2}$ respectively, and $\Gamma$ is $n_{1} \times n_{2}$. To see that (2) is a case of (1), take $m=2$ and

$$
A=\left(\begin{array}{ll}
G_{1} & G_{2} \\
G_{3} & G_{4}
\end{array}\right), \quad Y(t)=\left(\begin{array}{c}
\Gamma(t) \\
E_{n_{2}}
\end{array}\right) .
$$

For other related results see [4].

2. Let $X$ be $n \times n$ and such that

$$
X^{\prime}=A X
$$

The partitioning of $A$ induces a partitioning of $X$ into submatrices $X_{i j}(i, j=1, \cdots, k)$. Let $X_{m}=\operatorname{col}\left(X_{1 m} \cdots X_{k m}\right)$. Then

$$
X_{m}^{\prime}=A X_{m},
$$

and, at least formally,

$$
\left(X_{m m}^{-1}\right)^{\prime}=-X_{m m}^{-1} A_{m} X_{m m}^{-1} .
$$

Thus, if $X_{m m}^{-1}$ exists on some interval $I_{0} \subset I, X_{m} X_{m m}^{-1}$ is a solution on $I_{0}$ of (1). Further, if $Y$ is a solution of the differential equation of (1), $Y_{m}$ satisfies an equation of the form $P^{\prime}+H P=H$; thus from classical

Presented to the Society, January 25, 1961 under the title The cross-ratio property for the matrix Riccati equation; received by the editors June 13, 1960 and, in revised form, September 12, 1960. 
existence and uniqueness theorems (1) has a local existence and uniqueness theorem and $Y_{m}(t) \equiv E_{n_{m}}$. Gathering together these remarks, we have

THEOREM 1. Let $X(t)$ be the solution of (3) such that $X\left(t_{0}\right)=E_{n}$. Then the general solution near $t_{0}$ of (1) is $X(t) C\left(\sum_{i=1}^{k} X_{m i} C_{i}\right)^{-1}$, where $C$ is constant and arbitrary, $C=\operatorname{col}\left(C_{1} \cdots C_{k}\right), C_{i}$ is $n_{i} \times n_{m}$, and $C_{m}$ is nonsingular.

3. Now let $n=r k$ and $n_{i}=r(i=1, \cdots, k)$. We proceed to write the general solution of (1) in terms of solutions of (1) rather than solutions of (3). Let $U_{1}, \cdots, U_{k}$ denote solutions of (1); let $U=\left(U_{1} \cdots U_{k}\right)$; let $Z=\operatorname{diag}\left(Z_{j}\right)$, where $Z_{j}$ is $r \times r$ and

$$
Z_{j}^{\prime}=A_{m} U_{j} Z_{j} \quad(j=1, \cdots, k) .
$$

TheOREM 2. Let $U\left(t_{0}\right)$ and $Z\left(t_{0}\right)$ be nonsingular; let $C=\operatorname{col}\left(C_{j}\right)$, the $C_{j}$ being $r \times r$, constant, and arbitrary except that $\sum_{j=1}^{1} Z_{j}\left(t_{0}\right) C_{j}$ is nonsingular. Then the general solution of (1) can be written near $t_{0}$ as

$$
Y=U Z C \cdot\left(\sum_{j=1}^{k} U_{m j} Z_{j} C_{j}\right)^{-1} .
$$

Proof. It is easily verified that $U Z$ satisfies (3) and that, if $U\left(t_{0}\right)$ is nonsingular, $U(t)$ is nonsingular where it exists; the conclusion then follows.

Now let $U_{h i j}=U_{h i}-U_{h j}$ and $V_{h i j}=U_{h i j}^{-1}$. It is easily verified that $\left(U_{i}-U_{j}\right) V_{h i j}$ satisfies an equation of the form (1). Thus, if

$$
\left[\left(U_{i}-U_{q}\right) V_{h i q}-\left(U_{i}-U_{j}\right) V_{h i j}\right]_{t=t_{0}}=0,
$$

it is identically zero near $t_{0}$. Hence, if (8) is satisfied, $V_{h i j} U_{h i q}$ satisfies

$$
W^{\prime}=A_{m} U_{j} W-W A_{m} U_{q} .
$$

Now fix $q$; we may as well take $q=1$.

Theorem 3. Let $\left|U\left(t_{0}\right)\right| \neq 0$. Let $i=k+j-1(j=2, \cdots, k)$. Let $U_{i}$ be a solution of (1) such that, for some $r_{j}\left(1 \leqq r_{j} \leqq k, r_{j} \neq m\right), U_{r_{j} i j}$ and $U_{r_{j} i 1}$ are nonsingular at $t_{0}$ and (8) is satisfied for $q=1$ and $h=r_{j}$. Let $\left|Z_{1}\left(t_{0}\right)\right| \neq 0$. Then the general solution of (1) can be written in the form (7), where $Z_{j}=V_{r_{j} i j} U_{r_{j} i} Z_{1}(j=2, \cdots, k)$.

Proof. Note that $Z_{1}^{\prime}=A_{m} U_{1} Z_{1}$ and that $V_{r_{j} i j} U_{r_{j} i 1}$ satisfies (9) with $q=1$; then apply Theorem 2 .

We remark that, given $q, j$, and $h \neq k$, there does exist a set of initial values for the $U_{i}$ which satisfies (8). However, if for $U_{j}$ and 
$U_{p}(p \neq j)$ the same initial values for $U_{i}$ satisfy (8), there is a linear combination of columns of $U_{i}, U_{j}$, and $U_{p}$ which is zero. Thus, since $\left|U\left(t_{0}\right)\right| \neq 0$, each $U_{j}$ requires a distinct $U_{i}$. Thus, although we need not eliminate all of the $Z_{j}$ 's $(j=2, \cdots, k)$ in the general solution, we do need a distinct $U_{i}$ for each one we do eliminate.

For $k=2$ we have Theorem 5 of [3]. For $r=1$ and general $k$ we have a form for $Y$ different from that given in [2].

\section{BIBLIOGRAPHY}

1. A. Chiellini, Sui sistemi di Riccati, Rend. Sem. Fac. Sci. Univ. Cagliari vol. 18 (1948) pp. 44-58.

2. W. J. Coles, Linear and Riccati systems, Duke Math. J. vol. 22 (1955) pp. 333338.

3. J. J. Levin, On the matrix Riccati-equation, Proc. Amer. Math. Soc. vol. 10 (1959) pp. 519-524.

4. W. T. Reid, A matrix differential equation of Riccati-type, Amer. J. Math. vol. 68 (1946) pp. 237-246.

UNIVERSITY OF UTAH 